\title{
Dangerous Degree Evaluation System of Mine Debris Flow Based on IGA-BP
}

\author{
Xicheng Xue \\ Xi'an University of Science and Technology/College of Geology and Environment, Xi'an, China \\ xuexc331@163.com \\ Jisong Bi, Lingling Chen and Yan Chen \\ Xi' an University of Science and Technology/College of Geology and Environment, Xi'an, China \\ bijisong@163.com, ccblue2009@163.com,164574686@qq.com
}

\begin{abstract}
Taking the western Qinling Mountain, in the southern Shaanxi Province of china, as an example, based upon comprehensive analysis of geological data for 20 debris flow gullies, the author has put forward a series of indices system and has developed one evaluation system called “dangerous degree evaluation system of mine debris flow based on IGA-BP”. This system adopts Visual Basic 6.0 and Access technology to manage database, adopts immune genetic algorithm to optimize the hidden layer structure and network parameters of BP neural network and adopts sample model of mine debris flow whose dangerous degree has been known to realize the BP neural network evaluation of the debris flow risk which to be determined. The calculating results show that this evaluation method has high reliability and simplicity of operation, and it can make comprehensive evaluation precisely. The evaluation results have important guiding significance in the prevention and reduction mine debris flow.
\end{abstract}

Index Terms-Qinling, mine debris flow, dangerous degree evaluation system, immune genetic algorithm, artificial neural network

\section{INTRODUCTION}

1. Mine debris flow is a prominent environmental geological problem caused in the course of mine development and construction. The construction of mine and development of resources are often affected by the frequent occurrence and damage of debris flow. As a

Corresponding author, Jisong Bi. result, the dangerous degree evaluation of mine debris flow should be strengthened. The dangerous degree evaluation of mine debris flow is mainly contains the evaluation of the occur risk of debris flow disaster, the range and extend of the disaster as well as the loss of destroy ect.. The evaluation laid a solid foundation for recognizing the disaster situation of debris flow, making prevention policies as well as making plan, prevention and control measures for control regions. But the diversity and uncertainty of inducing factors of debris flow greatly increase the difficulty of the debris flow evaluation.

The western Qinling is taken as study object in this paper. It is located in the source of Jialing River and Han River, which is rich in metal mineral resources, such as $\mathrm{Pb}, \mathrm{Zn}, \mathrm{Au}$ and so on. These metals are concentrated in mineral accumulation areas such as Fengxian, Taibai ect.. Since the reform and open, various regions have been developing the mining economy positively by the right of their resource superiority. The mining development has made a significant contribution for the development of the local economy as well as the social progress. However, the mine debris flow occur frequently because of the high-strength exploit of mineral resources, the strong disturbance of geological environment caused by mining activities and the recklessly stacking of waste slag, rocks and tailings [1]. In August 1981, affected immediately by the strong rainstorm, large areas of debris flow occurred in western Qinling. With a large amount of waste slag, the debris flow buried a train of 
Honghuapu Station making bridges and roads blocked and destroyed by detritus, traffic suspended for 60 days, which made heavy losses for the national economy [2]. On September 18th, 2001, after a heavy rain, a mine debris flow occurred abruptly in qiantongshan mining area. The mountain slumping stuff in the upper reaches of the cleugh and the mine waste slag poured down, which buried the transport vehicles, suspended the traffic of the mining area and also caused heavy losses. As a result, the dangerous degree evaluation of mine debris flow should be put into effect as soon as possible in order to instruct the prevention and reduction of disaster of mine debris flow scientifically.

\section{The DESIGN PRINCIPLE OF IGA-BP EVALUATION MODEL}

\section{A. The Limits of Traditional Mathematical Evaluation Models}

The influencing factors of dangerous degree of mine debris flow are complicated and numerous. Assessing its dangerous degree scientifically applies important basis for the management planning and engineering design of mine debris flow. For the past few years, a lot of scholars have been carrying studies on the dangerous degree of mine debris flow from different perspectives, and have obtained plentiful achievements [3-8]. From the traditional methods like torrent classification, quantitative discrimination act to the later fuzzy comprehensive evaluation, mathematical methods mathematical methods are increasingly widely used in the evaluation of mine debris flow risk. While the superiority of those mathematical methods mentioned appear fully, their disadvantages are also exposed in practice, which has attracted increasing attention. Methods which were used earlier such as torrent classification, quantitative discrimination act are simple to operate, but the formulas themselves are in the status of semiquantitative "man-made scoring". There are more human experience and subjective effects in these methods. While fuzzy comprehensive evaluation has a strong ability of data expression. It can take different index weights into account and carry out comprehensive evaluation on multi-factors. But in practice, it is often difficult to determine the membership function and weight sets objectively.

\section{B. The Present of Evaluation Model Based on Immune Genetic Neural Network}

The dangerous degree of mine debris flow can be measured by a risk degree with a probability conception. This risk degree is valued in the closed interval of $[0,1]$. With the development of artificial intelligence technology and object-oriented programming techniques, it will be an inevitable trend to evaluate the dangerous degree of mine debris flow using artificial intelligence theory. At present, artificial neural network is being widely used because of its advantages of self-organization, self-learning, concertedness and high precision. The traditional methods need to determine membership function and weight sets artificially, besides they don't have self-learning, association and memory function. Comparatively speaking, the artificial neural network model can overcome these disadvantages perfectly, however, it still has its disadvantages. Given this, it lays foundation for the training of artificial neural network to make global optimization for hidden layer structure and network parameter using genetic algorithm base on immune theory (that is IGA model). Due to the immunological memory and self-regulation of IGA Model, the global search ability of genetic algorithm can be enhanced, and thus can avoid trapping into local solution. IGA Model also has the function of maintaining the diversity of antibodies which can enhance the global search ability, avoid premature convergence and insure a rapid convergence to the global optimum solution.

\section{The Basic Principle of BP Neural Network}

The artificial neural network is a kind of nonlinear mathematical model based on the theory of imitating the impulse conduction of human cranial nerve. It is a large-scale complex net system composed of numerous neurons by a certain topological structure. It is used to imitate the structure and action of human cranial nerve network [9]. The artificial neural network can learn knowledge from the experience of experts, summarize judge rules and solve problems through modifying evaluation model by itself as long as provided sufficient representative learning samples. According to the 
difference of its function, structure and learning manner, the artificial neural network can be divided into various models, among which the more mature one is multi-layer error back propagation neural network (BP neural network for short). This model is composed of input layer, output layer and one or many hidden layers. Each layer contains several neurons. Neurons of each layer connect those in other layers by full join while no connection with those in the same layers(Figure 1), which can realize the transformation of signal from input space to output space. Its information processing ability comes from the several compositions of simple nonlinear functions. And it is generally suitable for the forecast, pattern recognition and the approximation of nonlinear functions [10]. This model is widely used because of its high nonlinear dynamic handling ability and the self-learning, associate and memory functions of imitating human cranial nerve. But the determination of its hidden layer structure, the number of neurons, the initial threshold and the initial connection weight value is faced with the new problem of global optimization [11].

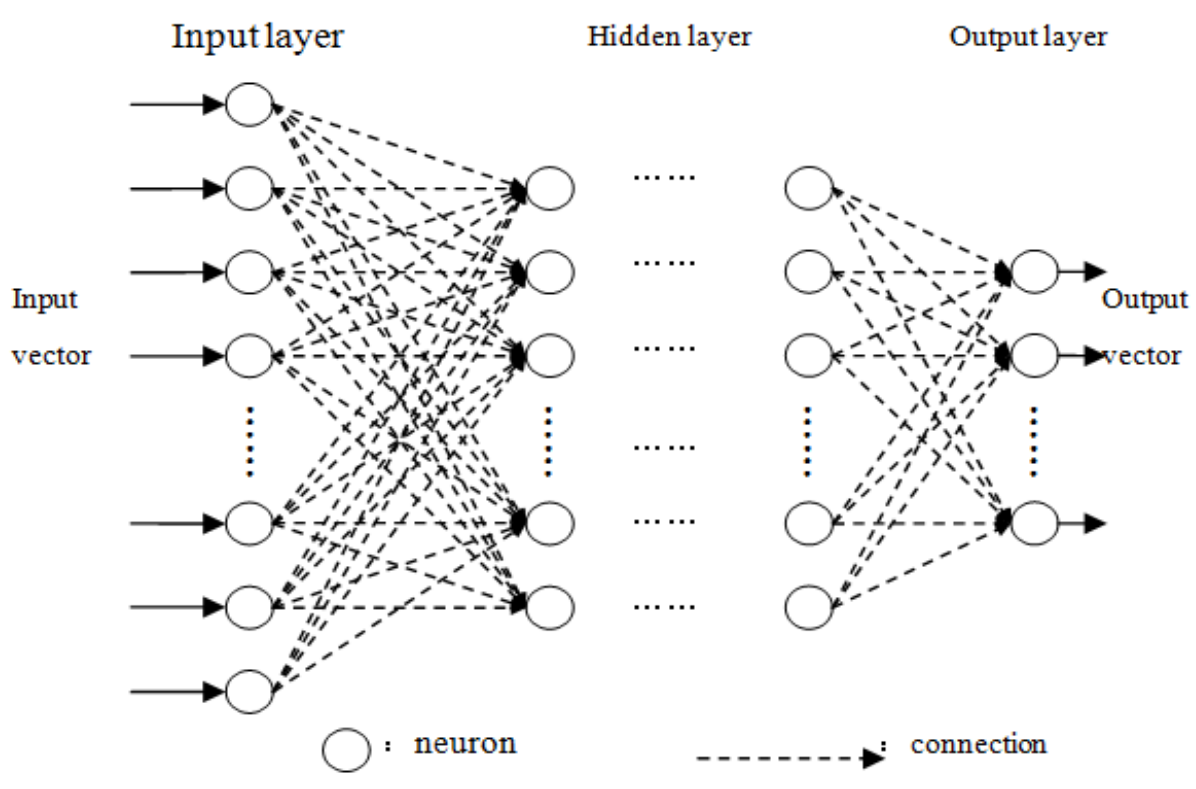

Figure 1 The structure sketch map of neural network

\section{The Basic Principle of Immune Genetic Algorithm}

Genetic Algorithm is a kind of random search algorithm taking example by natural selection and nature genetics mechanism in biosphere. It takes the objective function of practical problems to be solved corresponding to antigen while the solution of problems to antibody. It applies to the complex and nonlinear problems which are difficult to solve by traditional search algorithm. However, in practice, there are some problems to be discussed further [12-13]. For example, for monotonic functions or unimodal functions, they approach the optimal value rapidly at the beginning while converge slowly nearby the optimal value. For the optimization problems of multimodal functions, they always converge to local extremum rapidly. While the genetic algorithm
(Immunity Genetic Algorithm, or IGA for short), based on immune theory put forward in this paper, is an improvement of traditional genetic algorithm (GA). It retains the trait of GA of stochastic global parallel search, and also avoids premature convergence to a great extend in order to ensure the rapid convergence to the global optimal solution [14-15]. Immunity Genetic Algorithm overcome the too far or too slow convergence of traditional genetic algorithm, especially the premature of the rapid convergence to local extremum. What's more, this new algorithm can effectively improve the drawback of premature convergence and enhance the ability of global and local search and optimization to realize the global optimization of hidden layer structure, initial threshold and weight of artificial neural network [16], 
which will make it easy to operate the artificial neural network comprehensive evaluation model.

The specific process of immunity genetic algorithm is devised according to the certain chromosome coding scheme and immune operators, as is shown in Figure 2. The objective function and all kinds of evaluation indexes are input at first to act as antigens of immunity genetic algorithm. Then a set of initial antibodies (corresponding to candidate solutions) are produced randomly. While for the secondary response, it needs the help of the memory function of immune mechanism. Parts of the initial antigens are obtained through mnemon.
The good fitness and population distribution of the antibodies in mnemon can enhance the convergence rate. And then the fitness of every generation of antibodies are calculated with crossover and mutation carried out on these antibodies. Afterwards, the next generation of antibody groups is generated through updating strategy by the groups which are based on antibody concentration and affinity. End the whole algorithm till the end condition is satisfied. Finally, the results are substituted into BP neural network to carry out the network training and evaluation.

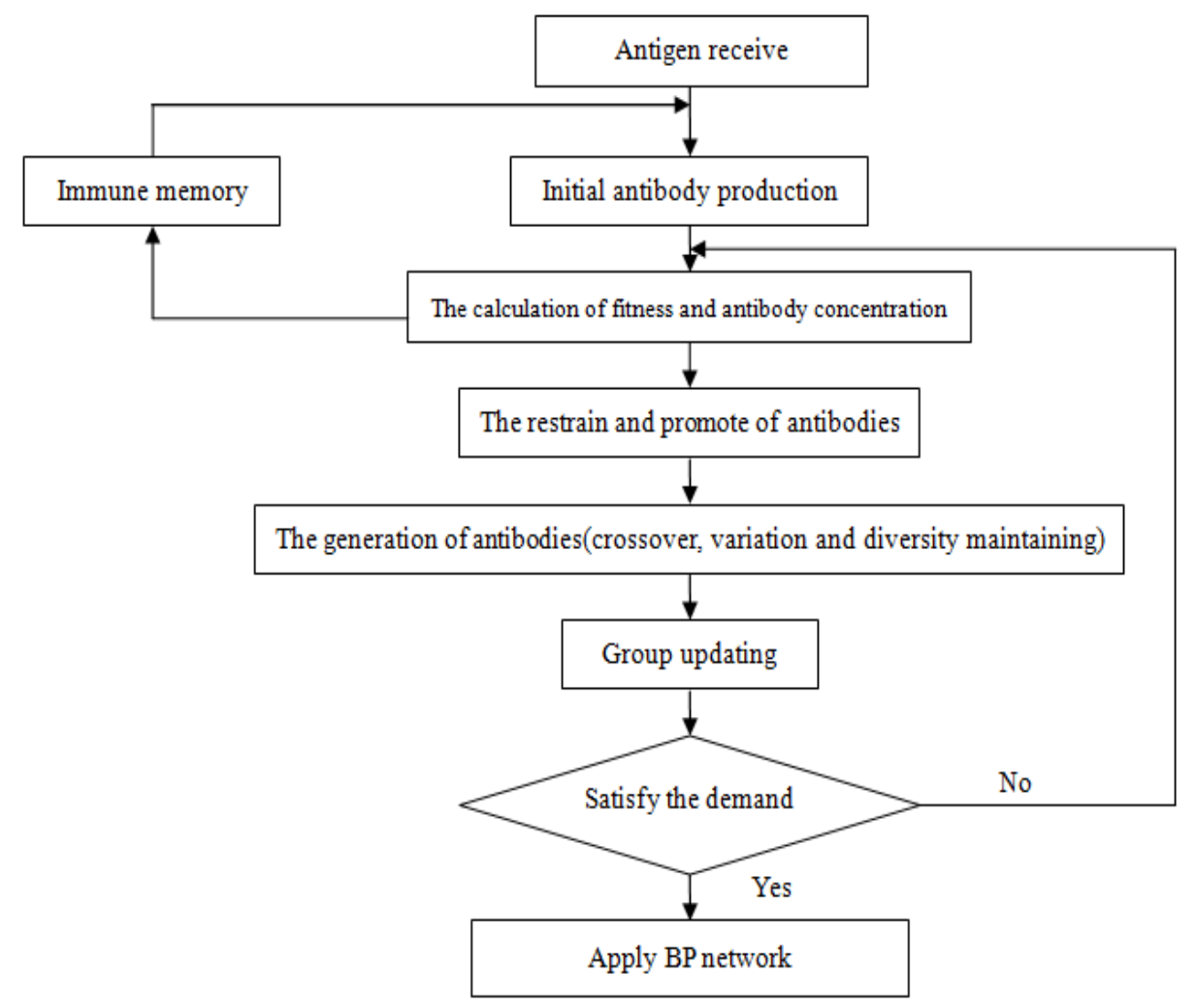

Figure 2 The flow chart of immunity genetic algorithm

\section{E. The Overall Design of The Syetem}

According to the overall designing thought and process of the system algorithm, "the dangerous degree evaluation system of mine debris flow based on IGA-BP” accomplished the design of the interface and the whole module of the system by taking object-oriented programming language Visual Basic 6.0 and Access database programming language as major development tool. This system fully absorbed a series of advantages of database technology, fuzzy mathematics, gray system, immune genetic algorithm and artificial neural network ect.. The system adopted the thought of object-oriented and human program design and finally realized the dangerous degree comprehensive evaluation of single debris flow gully, which laied a foundation for the control and prevention of debris flow. The functions of this system are shown in Figure 3. 


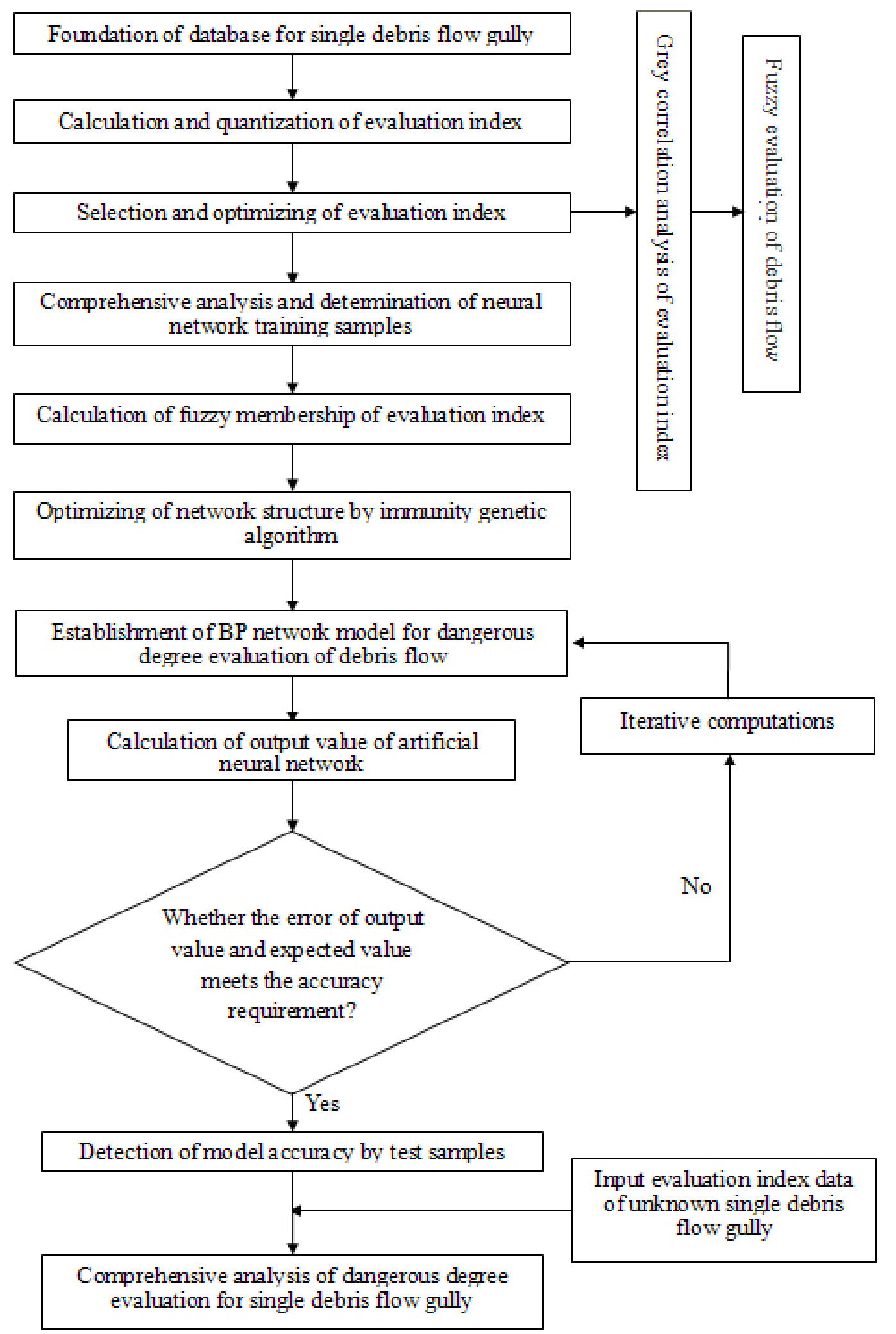

Figure 3 The overall design of the dangerous degree comprehensive evaluation system of debris flow 


\section{THE PROCESS OF COMPREHENSIVE EVALUATION} SYSTEM

Combine the immune genetic algorithm and artificial neural network mathematical model sufficiently. Then use the immune genetic algorithm to optimize the hidden layer structure and network parameters of BP neural network. Next train the BP neural network using the sample model of mine debris flow whose dangerous degree has been determined. And finally realize the BP network evaluation to the dangerous degree of mine debris flow which to be determined.

\section{A. Use IGA To Optimize The BP Network Structure}

(1) Input data: use "immune genetic algorithm module" to open the corresponding program interface. Click the button of "Yes" in the column of "input data", thus the data file of training samples and samples to be tested will be input automatically. The textbox corresponding to "the maximum number of input nodes" and "the maximum number of output nodes" separately show the number of input units and output units of selected training samples. Click the button of "Yes" and then come into the parameter setting column of immune genetic algorithm.

(2) Set parameters of immune genetic algorithm: input "population size", "the maximum number of hidden layers", "the maximum number of hidden nodes", "the maximum iterations", "the crossover rate of the first and the second layers", "the mutation probability of the first and the second layers", "the mutation probability of the third layer" and "concentration threshold" into the parameter setting column of immune genetic algorithm separately. In which, "population size" refers to the amount of antibodies in immune genetic algorithm; "the maximum number of hidden layers" refers to the maximum number of hidden layers which probably appear, no more than 4 in general; "the maximum number of hidden nodes" refers to the maximum number of hidden units which probably appear in single hidden layer, suggesting no more than two times of the maximum number of input nodes; "the maximum iterations" refers to the iterations till the end of immune genetic algorithm; "the crossover rate of the first and the second layers” refers to the crossover rate of antibody genes in the first and the second layers, and the number is valued in $(0,1)$, the larger the value, the more the crossover times, no more than 0.8 for suggestion; "the mutation probability of the first and the second layers" refers to the mutation probability of antibody genes in the first and the second layers, and the number is valued in $(0,1)$, the larger the value, the more the mutation times, and too high mutation probability may make the algorithm unstable, so the number is better no more than 0.2 ; "the mutation probability of the third layer" refers to the mutation probability of antibody genes in the third layer with its range in $(0,1)$, and no more than 0.6 for better; "concentration threshold" refers to the maximum limit of antibody concentration in immune genetic algorithm.

(3) Optimize the BP network structure: after setting the basic parameters of immune genetic algorithm, click the button of "optimize the network structure" and then start the immune genetic algorithm module. Though complicated iterative computation, obtain globally optimized hidden layer structure and network parameters of BP neural network. The results of immune genetic algorithm show that, for fixed training sample data and number of input and output neurons, the optimal hidden layer structure of BP neural network can be described as follows: 1 hidden layer and 8 hidden layer neurons. Accordingly the optimal hidden layer structure of BP neural network is determined as type of 33-8-3.

\section{B. The Training of BP Neural Network}

The training of artificial neural network is the process of self-learning and self- adapting. The training is the core of the treatment of artificial neural network, and it consists of the process of mode order transmission, error back propagation, memory training and network converging. Use "artificial neural network module" to open the training interface of BP neural network (Figure 4). Click the button of "start training" to start the artificial neural network module. The program will carry on network training and self-learning using the training samples and expected output value determined before. The actual output values approach to its expected output value gradually and as a result the network errors get corrected constantly until the overall error is below the overall error level. 


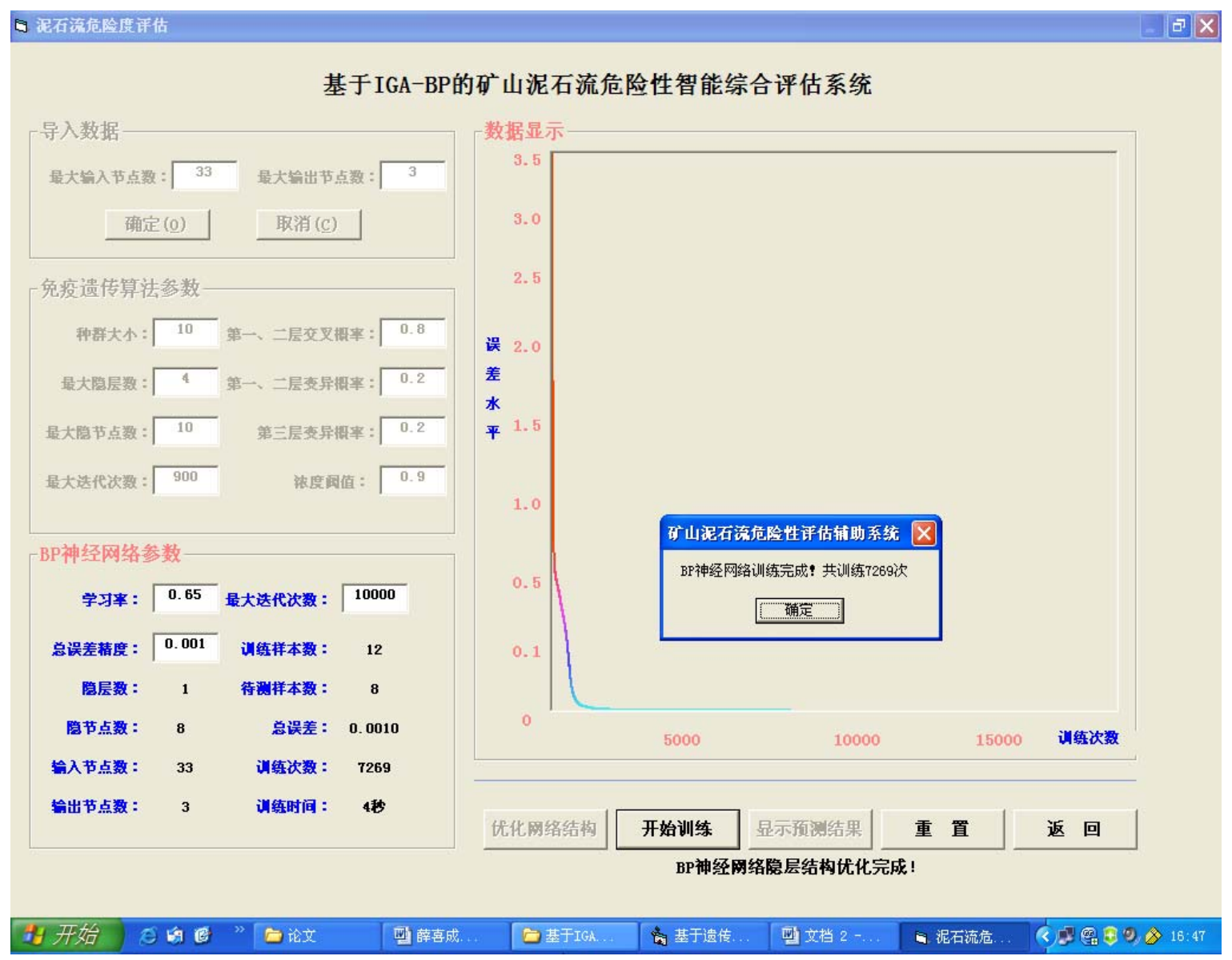

Figure 4 Main interface of software system

This interface is divided into two parts. The dynamic of the right part shows the change curve of the training overall error of artificial neural network. The abscissa of this curve represents the network training times, and the ordinate refers to the overall error of network. And the curve clearly show that the overall error of network decreased with the increase of its training times. While the left part shows the parameters related to the training of artificial neural network, including the number of hidden layer neurons, the learning rate of neurons, momentum factors of neurons, overall error level, the number of training samples, the number of input neurons, the number of output neurons, overall error, training times ,training time and so on.

When the training of $\mathrm{BP}$ neural network is over, mature weights and threshold will be stored in BP neural network in order to be used by comprehensive evaluation program module.

\section{The Distinction Examination of BP Neural Network} Training Samples
After the training of neural network mentioned above, neural network models which had been trained were used to do distinction examination on 24 training sample data [17]. As a result, 3 output neuron values, absolute error, relative error and the types of dangerous degree of mine debris flow were obtained (TABLE I ). The results show that for the network expectant output values of training samples, the average absolute error of the whole training samples is 0.015 , the average relative error is only $5.1 \%$, and no erroneous judgement occurred, which indicates that the BP intelligent comprehensive evaluation models has high fitness to the evaluation of training samples. So the BP neural network can be used to make intelligent comprehensive evaluation to the mine debris flow risk. Of course, if the error of comprehensive evaluation of BP neural network is too large, it is necessary to return to the BP neural network training module to train again or exam the advantages and disadvantages of the training samples. 
TABLE I

DISTINCTION EXAMINATION EFFECT OF BP NEURAL NETWORK TRAINING SAMPLE

\begin{tabular}{|c|c|c|c|c|c|c|c|}
\hline Number & Name & Neuron 1 & Neuron 2 & Neuron 3 & Absolute error & Relative error & Dangerous degree \\
\hline N1 & Daheigou & 0.073 & 0.952 & 0.006 & 0.048 & $4.8 \%$ & Subsidiary risk \\
\hline N2 & Jiashangou & 0.073 & 0.975 & 0.006 & 0.025 & $2.5 \%$ & Subsidiary risk \\
\hline N3 & Zhaizigou & 0.016 & 0.970 & 0.011 & 0.030 & $3.0 \%$ & Subsidiary risk \\
\hline N4 & Shuijingwan & 0.057 & 0.025 & 0.959 & 0.041 & $4.1 \%$ & Risk \\
\hline N5 & Guanmengou & 0.073 & 0.031 & 0.904 & 0.096 & $9.6 \%$ & Risk \\
\hline N6 & Yinmusigou & 0.038 & 0.019 & 0.930 & 0.070 & $7.0 \%$ & Risk \\
\hline N7 & baguagou & 0.031 & 0.962 & 0.015 & 0.038 & $3.8 \%$ & Subsidiary risk \\
\hline N8 & Hetaogou & 0.053 & 0.011 & 0.935 & 0.065 & $6.5 \%$ & Risk \\
\hline N9 & Momengou & 0.027 & 0.948 & 0.065 & 0.052 & $5.2 \%$ & Subsidiary risk \\
\hline N10 & Mahuanggou & 0.025 & 0.937 & 0.041 & 0.063 & $6.3 \%$ & Subsidiary risk \\
\hline N11 & Changgou & 0.065 & 0.897 & 0.010 & 0.103 & $10.3 \%$ & Subsidiary risk \\
\hline N12 & Erlihe & 0.003 & 0.034 & 0.921 & 0.079 & $7.9 \%$ & Risk \\
\hline N13 & Xiaodongwan & 0.001 & 0.936 & 0.036 & 0.064 & $6.4 \%$ & Subsidiary risk \\
\hline N14 & Lishugou & 0.024 & 0.075 & 0.958 & 0.042 & $4.2 \%$ & Risk \\
\hline N15 & Shuijinggou & 0.056 & 0.042 & 0.976 & 0.024 & $2.4 \%$ & Risk \\
\hline N16 & Guoansi & 0.008 & 0.990 & 0.076 & 0.01 & $1.0 \%$ & Subsidiary risk \\
\hline N17 & Lijiagou & 0.039 & 0.930 & 0.009 & 0.07 & $7.0 \%$ & Subsidiary risk \\
\hline N18 & Zhujiaba & 0.067 & 0.973 & 0.031 & 0.027 & $2.7 \%$ & Subsidiary risk \\
\hline N19 & Dengjiatai & 0.039 & 0.920 & 0.007 & 0.08 & $8.0 \%$ & Subsidiary risk \\
\hline N20 & Wujiagou & 0.978 & 0.034 & 0.017 & 0.022 & $2.2 \%$ & No risk \\
\hline N21 & Guangou & 0.987 & 0.013 & 0.038 & 0.013 & $1.3 \%$ & No risk \\
\hline N22 & Meigou & 0.962 & 0.036 & 0.075 & 0.038 & $3.8 \%$ & No risk \\
\hline N25 & Yindongliang & 0.023 & 0.040 & 0.968 & 0.032 & $3.2 \%$ & Risk \\
\hline N28 & Jiangjiagou & 0.067 & 0.023 & 0.915 & 0.085 & $8.5 \%$ & Risk \\
\hline verage value & & & & & 0.051 & $5.1 \%$ & \\
\hline
\end{tabular}

D. Comprehensive Evaluation of Dangerous Degree of Mine Debris Flow

The evaluation of unknown mine debris flow risk is the final task. On the basis of the accomplishment of the training and distinction examination of artificial neural network, users can use BP network which has been trained to evaluate the dangerous degree of objective mine debris flow by comprehensive evaluation modules. At the same time, 3 output neuron values are obtained from which we can determine the different types of the mine debris flow risk (TABLE II). 
TABLE II

INTELLIGENT EVALUATION RESULT OF MINE DEBRIS FLOW RISK

\begin{tabular}{|c|c|c|c|c|c|}
\hline \multirow{2}{*}{ Number } & Debris flow & Neuron 1 & Neuron 2 & Neuron 3 & Results of comprehensive evaluation \\
\hline N23 & Tangjiagou & 0.995 & 0.025 & 0.012 & No risk \\
\hline N24 & Tancaogou & 0.987 & 0.051 & 0.009 & No risk \\
\hline N26 & Sigou & 0.012 & 0.061 & 0.985 & Risk \\
\hline N27 & Mutonggou & 0.023 & 0.941 & 0.077 & Subsidiary risk \\
\hline N29 & Erdaogou & 0.005 & 0.043 & 0.967 & Risk \\
\hline N30 & Sandaogou & 0.021 & 0.020 & 0.990 & Risk \\
\hline N31 & Sidaogou & 0.007 & 0.032 & 0.964 & Subsidiary risk \\
\hline N32 & Santaishangou & 0.027 & 0.982 & 0.045 & Subsidiary risk \\
\hline N33 & Shangpinggou & 0.058 & 0.992 & 0.003 & Subsidiary risk \\
\hline N34 & Hougou & 0.086 & 0.965 & 0.027 & R \\
\hline
\end{tabular}

\section{IV.CONCLUSION}

"IGA-BP neural network" is a kind of nonlinear mathematical method. It is a new kind of composite algorithm which concentrated and imitated three pillars systems of human life. This method doesn't need precondition and constraint condition, and no necessary to built rigorous evaluation mathematical model in advance. The model will accomplish the network optimizing, self-learning and self-training as long as provided high quantity learning samples. It will summarize a set of evaluation plans instead of storing in the BP neural network connection weights and threshold in the way of nonlinear quantization relation and implicit expression.

"The dangerous degree evaluation system of mine debris flow based on IGA-BP” fully absorbed a series of advantages of mathematical models such as database technology, fuzzy mathematics, gray system, immune genetic algorithm, artificial neural network and so on. It realized immune genetic algorithm treatment and $\mathrm{BP}$ neural network training by adopting the thought of object-oriented and human program design. It also realized the objective comprehensive evaluation of dangerous degree of mine debris flow by making distinction examination to BP neural network. At the same time the accuracy and reliability of special software system have been proved. Before the use of software system, it requires geological professionals to observe at spot and collect related raw data of terrain, physiognomy, lithological character, structure, mine development and so on. Then set the basic data base of mine debris flow and select network training samples. The software modules will automatically realize index quantification processing, immune genetic calculation, artificial neural network training and the comprehensive evaluation of the mine debris flow risk. The results of living examples show that the immune genetic neural network put forward in this paper is not only intelligentized highly and easy to operate, but also more accurate and reliable for the comprehensive evaluation of dangerous degree of mine debris flow.

\section{ACKNOWLEDGMENT}

Thanks to the subsidy of Shaanxi Nature Science Fund Project (SJ08-ZT08-3).

\section{REFERENCES}

[1] HOU Enke, HAO Zhucheng, WANG Xiangyang, “The present state of geological environment and restoring strategy in lead-zinc mining areas in Feng Xian," Nonferrous Metals(Mining), No. 5, pp.12-14, 2001.

[2] CUI Peng, WEI Fangqiang, XIE Hong etc, "Debris flow and disaster reduction strategies in western china," Quaternary sciences, Vol. 23, No. 2, pp. 142-151. 2003.

[3] LIU xilin, TANG Chuan, "The mudslide risk evaluation," Beijing: Science publisher, 1995. 
[4] LIU xilin,TANG Chuan, ZHANG Songlin.Quantitaive judgment on the debris flow risk degree [J].The disaster learn,1993,8(2):1-71.

[5] WEI yongming, XIE Youyu, WU Yongqiu, “Applications of relativity analysis method and fuzzy synthetical assessment method in classification of dangerous degree of debris flow," Natural disaster college journal, Vol. 7, No. 2, pp. 109-117, 1998.

[6] LIU Yongjiang, HU Houtian, BAI Zhiyong, "Mudslide dangerous degree the nerve network method for evaluate," The geology and Prospecting, Vol. 37, No. 2, pp.84-87, 2001.

[7] KUANG Lehong, LIU Baochen, YAO Jingcheng, "Research on Regionalization of Debris Flow Risk Degreewith Fuzzy and Extension Method," Catastrophology, Vol. 21, No.1, pp. 68-72, 2006.

[8] LIU Jinfeng, OU Guoqiang, "New opinion on debris flows hazard assessment," The geology disaster and environmental protection, Vol. 15, No. 1, pp. 5-8, 2004.

[9] XIA Yucheng, CHEN Lianwu, XUE Xi-cheng, "Geoscience information numeralization outline," Xi'an: Shaanxi Science and Technology Press, 2003.

[10] HAN liqun, "The Artificial Neural Network," Beijing: Beijing University of Posts and Telecommunications Press, 2006.

[11] ZHAO Yuan, LIU Xilin, "Application of Ann to Risk Assessment on Debris Flow," Journal of Geological, Hazards and Environment Preservation, Vol. 16, No. 2, pp.135-138, Jun. 2005.

[12] Soon Thiam Khu, "Genetic programming and its application in real-time runoff forecasting," Journal of the American Water Resources Association, Vol. 23, No. 2, pp. 439-450,2001.

[13] J G Na, "Adaptive optimization of fed-batch culture of yeast by using genetic algorithms," Bioprocess and biosystems engineering, No. 4, pp. 299-308, 2002.

[14] LI Dake, PAN Zhimin, "Easy Modeling of Genetic Algorithm,” Journal of Kashgar Teachers College, Vol. 26, No. 6, pp.11-13, Nov. 2005.

[15] YANG Jianguo, WENG Shanyong, and ZHAO Hong, “An Optimized BP Network Model Using Genetic Algorithm for Predicting the Ignition-Stability Index of Pulverized Coal,” Journal of Power Engineering, Vol. 26, No. 1, pp.81-83, Feb. 2006.

[16] ZHOU Ming, SUN Shudong, "Genetic Algorithms: Theory and Applications,” Beijing: National Defense Industry Press, 1999.

[17] Zhu A X, "Mapping soil landscape as spatial continua: the neural network approach,” Water Resources Research, Vol. 36, No. 3, pp.663-677, 2000.

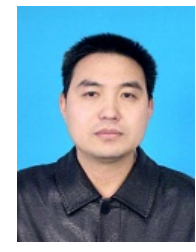

Xicheng Xue was born in Ruicheng, Shanxi Province, P.R. China in 1968, received the B.E. degree in the field of Coal Geology and Exploration in 1991 from Shanxi Mining Institute, the M.E. degree in 1994 from Xi'an Mining Institute, and the D.E. degree in the field of Mining Engineering in 2008 from Xi'an University of Science and Technology. He is a professor in environmental geology and computer application now.

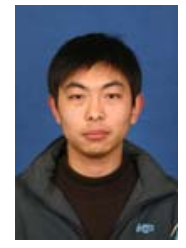

Jisong Bi was born in TsingTao, Shandong Province, P.R. China in 1986, received the B.S. degree in the field of Geography in 2009 from University of Jinan, He is M.E. candidate in the Environment Engineering of Xi'an University of Science and Technology, and is expected to graduate in June 2012. He mainly research on geological disaster and computer applications.

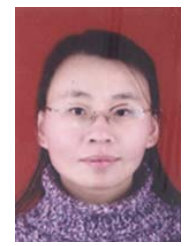

Lingling Chen was born in Laiwu, Shandong Province, P.R. China in 1986, received the B.S. degree in the field of Environment Science in 2009 from Shandong Normal University. She is M.E. candidate in the Environment Science of Xi'an University of Science and Technology, and is expected to graduate in June 2012. She mainly research on heavy metal pollution and repair of soil.

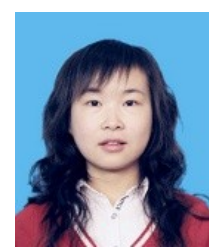

Yan Chen was born in Korla, Xinjiang Province, P.R. China in 1986, received the B.E. degree in the field of Environment science in 2009, and she is M.E. candidate in the Environment engineering of $\mathrm{Xi}^{\prime}$ an University of Science and Technology, and is expected to graduate in June 2012. She mainly research on mine environmental restoration and management. 